\title{
Organising Committee
}

\section{The 43rd Annual Meeting of the European Society for Blood and Marrow Transplantation: Organising Committee}

\author{
26-29 March 2017 • Marseille, France \\ Copyright: Modified and published with permission from EBMT, http://www.ebmt2017.org/
}

Sponsorship Statement: Publication of this supplement is sponsored by the European Society for Blood and Marrow Transplantation.

Christian Chabannon, Congress President

Didier Blaise, Congress Scientific Committee Co-Chair

Gérard Michel, Congress Scientific Committee Co-Chair

\section{Honorary Congress Presidents}

Dominique Maraninchi (Marseille)

\section{Local Organising Committee}

Jean-Marie Boher (IPC)

Jean-Paul Borg (Centre de Recherches en Cancérologie

de Marseille, CRCM)

Reda Bouabdallah (IPC)

Boris Calmels (Société Francophone de Greffe de Moelle \& de Thérapie Cellulaire, SFGM-TC \& IPC)

Guillaume Cartron (Centre Hospitalier et Universitaire de Montpellier, CHU-M)

Luca Castagna (IPC)

Hervé Chambost (AP-HM)

Jacques Chiaroni (Etablissement Français du Sang

Alpes-Méditerranée, EFS-AM, Marseille)

Carole Coze (AP-HM)

John de Vos (SFGM-TC \& CHU-M)

Raynier Devillier (IPC)

Catherine Faucher (IPC)

Cyril Fauriat (CRCM)

Nathalie Fegueux (CHU-M)

François Feron (Aix-Marseille Université, AMU)

Sabine Fürst (IPC)

Claire Galambrun (AP-HM)

Anthony Goncalves (IPC)

Angéla Granata (IPC)

Samia Harbi (IPC)

Anne-Gaelle Le Corroller (Inserm UMR 912/SESSTIM)

Fawzeh Legrand (IPC)

Claude Lemarie (IPC)

Jeremy Magalon (AP-HM)

Dominique Maraninchi (IPC)

Claude Mawas (CRCM)

Bilal Mohty (IPC)

Bertrand Nadel (Centre d'Immunologie de Marseille-Luminy, CIML \& Cancéropôle PACA, Marseille)

Daniel Olive (CRCM)

Claire Oudin (AP-HM)

Régis Peffault de Latour (SFGM-TC)
Christophe Picard (EFS-AM)

Pierre Simon Rohrlich (SFGM-TC \& Nice University Hospital, Centre Hospitalier et Universitaire de Nice, CHUN)

Florence Sabatier (AP-HM)

Jean-Marc Schiano de Collela (IPC)

Anne-Marie Stoppa (IPC)

Isabelle Thuret (AP-HM)

Sophie Ugolini (CIML)

Julie Veran (AP-HM)

Norbert Vey (IPC)

Patrice Viens (IPC)

Eric Vivier (CIML \& AP-HM)

Pierre-Jean Weiller (IPC)

Ibrahim Yakoub-Agha (SFGM-TC)

\section{Local Nurses Organising Committee}

Marie Angeli, Chair

Laurence Caymaris (IPC)

Carine Malenfant (IPC)

Anne-Claire Rampal (IPC)

\section{EBMT Board and Working Party Chairs}

Mohamad Mohty, President

Rafael Duarte, Secretary

Jürgen Kuball, Treasurer

Andreu Gusi, Executive Director

Nicolaus Kröger, Scientific Council Chair

Arnon Nagler, Scientific Council Co-Chair

Carlo Dufour, Scientific Council Education Representative

Chiara Bonini, Scientific Council Registry Representative

Aleksandra Babic, President Nurses Group

Christian Chabannon, EBMT 2017 Congress President

Arnon Nagler, Acute Leukemia

Carlo Dufour, Severe Aplastic Anaemia

John Snowden, Autoimmune Diseases

Nicolaus Kröger, Chronic Malignancies

Chiara Bonini, Cellular Therapy \& Immunobiology

Jan Styczynski, Infectious Diseases

Rafael Duarte, Complications and Quality of Life

Silvia Montoto, Lymphoma

Andrew Gennery, Inborn Errors

Peter Bader, Paediatric Diseases

Francesco Lanza, Solid Tumours 


\section{EBMT Nurses Group \& Scientific Committee}

Aleksandra Babic, Nurses Group President John Murray, Nurses Group President-Elect

Marie Angeli, EBMT 2017 local nurse

Caroline Bompoint

Ilona Siljander

Kathy Goris

Letizia Galgano

Simone van der Linden

Elsa Oliveira, EBMT 2018 local nurse

\section{Data Management Group}

Carmen Ruiz

Shelley Hewerdine

Khrystyna Valkiv
Statistics Group

Richard Szydlo

\section{Quality Management Group}

Renza Monteleone, Chair

Phuong Huynh

Ilknur Kozanoglu

Mara Magri

Ines Pronk

Nick van Sinderen

Anne Emmet 